PROCEEDINGS OF THE

AMERICAN MATHEMATICAL SOCIETY

Volume 133, Number 10, Pages 3057-3064

S 0002-9939(05)07882-2

Article electronically published on April 19, 2005

\title{
BOUNDS ON THE NUMBER OF HOLOMORPHIC MAPS OF COMPACT RIEMANN SURFACES
}

\author{
MASAHARU TANABE \\ (Communicated by Juha M. Heinonen)
}

\begin{abstract}
We give bounds on the number of nonconstant holomorphic maps of compact Riemann surfaces of genera $>1$.
\end{abstract}

\section{INTRODUCTION}

Let $X$ be a compact Riemann surface of genus $g(>1)$. De Franchis $[\mathrm{F}]$ stated the following:

Theorem of de Franchis. (a) For a fixed compact Riemann surface $Y$ of genus $>1$, the number of nonconstant holomorphic maps $X \rightarrow Y$ is finite.

(b) There are only finitely many compact Riemann surfaces $Y_{i}$ of genus $>1$ which admit a nonconstant holomorphic map from $X$.

The second statement (b) is often attributed to Severi. After knowing the finiteness of maps, we may ask if there exists an upper bound depending only on some topological invariant, for example, the genus $g$. Related to the statement (a), Martens [Mr1] showed if one fixes a Riemann surface $Y$, then the number of all nonconstant holomorphic maps from $X$ to $Y$ is less than $(c g)^{2 g^{2}}$ for some constant $c>1$ independent of $g$. Recently, the author [T2] showed that the bound is smaller than $(c g)^{2 g}$ for some constant $c$. Now, we consider a bound for holomorphic maps when $Y$ is not fixed, that is, we estimate the number of all nonconstant holomorphic maps from $X$ to other Riemann surfaces. Let $f_{i}: X \rightarrow Y_{i}$ be nonconstant holomorphic maps for $i=1,2$. We say that $f_{1}$ and $f_{2}$ are isomorphic if and only if there is a conformal map $h: Y_{1} \rightarrow Y_{2}$ such that $h \circ f_{1}=f_{2}$. Let $\mathcal{I}_{\gamma}(X)$ denote the set of all isomorphic classes of nonconstant holomorphic maps into compact Riemann surfaces of genus $\gamma>1$, and denote $\mathcal{I}(X)=\bigcup_{g>\gamma>1} \mathcal{I}_{\gamma}(X)$. By the theorem of de Franchis, we see that $\sharp \mathcal{I}(X)$ is finite. In 1983 Howard and Sommese $\mathrm{H}$-S first showed that there is a bound on $\sharp \mathcal{I}(X)$ depending only on $g$. In 1986 Kani $[\mathrm{K}$ gave a bound of morphisms from a smooth projective curve defined over a field $\mathbb{K}$, depending only on the genus of the curve. In particular, when $\mathbb{K}=\mathbb{C}$, he showed that

$$
\sharp \mathcal{I}(X)<(g-1) 2^{2 g^{2}-2}\left(2^{2 g^{2}-1}-1\right),
$$

Received by the editors March 26, 2002 and, in revised form, June 2, 2004.

2000 Mathematics Subject Classification. Primary 30F30.

The author's research was partially supported by the Japan-U.S. Cooperative Science Program.

(C)2005 American Mathematical Society Reverts to public domain 28 years from publication 
and in 1990 Alzati and Pirola $\mathrm{A}-\mathrm{P}$ gave a better bound

$$
\sharp \mathcal{I}(X)<\exp \left\{(4 / 3)\left(g^{2}-1\right) \log 3+\left[\log _{2} g\right] \log (84 g)+\log (12 \sqrt{2})\right\} .
$$

Here, we will improve them.

Theorem. Let $X$ be a compact Riemann surface of genus $g>1$. Then the number $\sharp \mathcal{I}(X)$ satisfies

$$
\sharp \mathcal{I}(X)<(2 g)^{4 g} \times 2^{2 g-3} \times(2 g-1)^{g-1}(2 g-3)(g-2)(g-1) .
$$

We will show this by estimating the possible number of pull backs of harmonic differentials in $H^{1}\left(Y_{i}, \mathbb{Z}\right)$. We may see that $H^{1}\left(Y_{i}, \mathbb{Z}\right)$ is the lattice of the dual Jacobian variety $\widehat{\mathrm{J}\left(Y_{i}\right)}$. Thus, we will use theory of homomorphisms of Jacobians and theory of lattices from the geometry of numbers.

Let

$$
M(g)=\max _{X}\{\sharp \mathcal{I}(X)\},
$$

where the maximum is taken over all Riemann surfaces $X$ of genus $g$. It is an interesting problem to determine the exact rate of growth of $M(g)$. By the Theorem, we see

$$
M(g) \leq(c g)^{5 g}
$$

for some constant $c$, while all the previously given ones (by Kani and AlzatiPirola) guarantee $M(g) \leq c^{g^{2}}$. On the other hand, Kani $[\mathrm{K}]$ also constructed a sequence $F_{1}, F_{2}, \ldots, F_{n}, \ldots$ of function fields over $\mathbb{K}$ of genus $g_{F_{1}}<g_{F_{2}}<\ldots<$ $g_{F_{n}}<\ldots$, such that the number of separable subfields of $F_{n} / \mathbb{K}$ is larger than $\exp \left(c\left(\log \left(g_{F_{n}}\right)\right)^{2}\right)$ for some constant $c>0$ (independent of $\left.n\right)$. This implies that $M(g)$ cannot be bounded by any polynomial in $g$.

\section{Notation And Lemmata}

In this paper, all of the Riemann surfaces will be compact and of genera greater than 1. First, we recall some notions from complex tori. Let $V$ be a complex vector space and $\Gamma$ a lattice in $V$. The quotient $T=V / \Gamma$ is called a complex torus. Denote by $\widehat{T}=V^{*} / \widehat{\Gamma}$ the dual where $V^{*}$ is the space of $\mathbb{C}$-antilinear functionals on $V$ and $\widehat{\Gamma}=\left\{l \in V^{*}: \operatorname{Im} l(\Gamma) \subseteq \mathbb{Z}\right\}$ is the dual lattice of $\Gamma$. Let $\mathfrak{f}$ be a homomorphism between two complex tori $T=V / \Gamma$ and $T^{\prime}=V^{\prime} / \Gamma^{\prime}$. Then, there is a unique $\mathbb{C}$-linear map $F: V \rightarrow V^{\prime}$ with $F(\Gamma) \subseteq \Gamma^{\prime}$ inducing $\mathfrak{f}$. We call $F$ the analytic representation of $\mathfrak{f}$, and the restriction $\left.F\right|_{\Gamma}$ the rational representation of $\mathfrak{f}$. For the analytic representation $F: V \rightarrow V^{\prime}$ of a homomorphism $f: T \rightarrow T^{\prime}$, the dual map ${ }^{t} F: V^{\prime *} \rightarrow V^{*}$ associating to an antilinear functional $l \in V^{\prime *}$ the antilinear functional $l \circ F \in V^{*}$ induces a homomorphism ${ }^{t} \mathfrak{f}: \widehat{T}^{\prime} \rightarrow \widehat{T}$, since ${ }^{t} F\left(\widehat{\Gamma^{\prime}}\right) \subseteq \widehat{\Gamma}$. We call ${ }^{t} \mathfrak{f}$ the dual map of $\mathfrak{f}$. Let $X$ and $Y$ be compact Riemann surfaces of genera $g$ and $\gamma$, respectively. Denote by $\mathcal{H}$ the space of holomorphic differentials on $X$. Set $\Omega=\operatorname{Hom}(\mathcal{H}, \mathbb{C})$. The Jacobian variety $\mathrm{J}(X):=\Omega / H_{1}(X, \mathbb{Z})$ is a complex torus of dimension $g$, and considering $\overline{\mathcal{H}}$ of $\mathbb{C}$-antilinear forms on $\Omega$, we will denote by $\widehat{\mathrm{J}(X)}=\overline{\mathcal{H}} / H^{1}(X, \mathbb{Z})$ the dual. In order to describe $\mathrm{J}(X)$ in terms of period matrices, choose basis $\lambda_{1}, \ldots, \lambda_{2 g}$ of $H_{1}(X, \mathbb{Z})$ and $\omega_{1}, \ldots, \omega_{g}$ of $\mathcal{H}$. Let $l_{1}, \ldots, l_{g}$ be the basis of $\Omega$ dual to $\omega_{1}, \ldots, \omega_{g}$, i.e., $l_{i}\left(\omega_{j}\right)=\delta_{i j}$ (Cronecker's delta). Considering 
$\lambda_{j}$ as a linear form on $\mathcal{H}$, we have $\lambda_{j}=\sum_{k=1}^{g}\left(\int_{\lambda_{j}} \omega_{k}\right) l_{k}$ for $j=1, \ldots, 2 g$. Hence

$$
\Pi_{X}=\left(\begin{array}{ccc}
\int_{\lambda_{1}} \omega_{1} & \cdots & \int_{\lambda_{2 g}} \omega_{1} \\
\vdots & & \vdots \\
\int_{\lambda_{1}} \omega_{g} & \cdots & \int_{\lambda_{2 g}} \omega_{g}
\end{array}\right)
$$

is a period matrix for $\mathrm{J}(X)$ with respect to these bases. Similarly, we define $\mathrm{J}(Y)$, $\widehat{\mathrm{J}(Y)}$ and $\Pi_{Y}$ for $Y$. Let $\mathfrak{f}: \mathrm{J}(X) \rightarrow \mathrm{J}(Y)$ be a homomorphism. In terms of matrices, $\mathfrak{f}$ can be expressed as

$$
A \Pi_{X}=\Pi_{Y} M
$$

with $A \in M(\gamma, g ; \mathbb{C}), M \in M(2 \gamma, 2 g ; \mathbb{Z})$ (we denote by $M(m, n ; K)$ the set of $m \times n$ matrices with $K$-coefficients). Conversely, if there are matrices $A \in M(\gamma, g ; \mathbb{C})$ and $M \in M(2 \gamma, 2 g ; \mathbb{Z})$ such that $A \Pi_{X}=\Pi_{Y} M$, then these matrices are matrix representations of some homomorphism $\mathrm{J}(X) \rightarrow \mathrm{J}(Y)$. We also call $A$ the analytic representation of $\mathfrak{f}$, and $M$ the rational representation of $\mathfrak{f}$ (with respect to the bases). There is a canonical principal polarization on $\mathrm{J}(X)$. Fix a homology basis $\lambda_{1}, \ldots, \lambda_{2 g}$ of $H_{1}(X, \mathbb{Z})$ with a intersection matrix

$$
J=\left(\begin{array}{cc}
0 & I \\
-I & 0
\end{array}\right)
$$

where each entry is $g \times g$ sized. Considered as a $\mathbb{R}$-vector space, $\lambda_{1}, \ldots, \lambda_{2 g}$ is a basis for $\Omega$. Denote by $E$ the alternating form on $\Omega$ with the matrix $J=\left(\begin{array}{cc}0 & I \\ -I & 0\end{array}\right)$ with respect to the basis $\lambda_{1}, \ldots, \lambda_{2 g}$ for $\Omega$ and define a hermitian form $H: \Omega \times \Omega \rightarrow \mathbb{C}$ by

$$
H(u, v)=E(i u, v)+i E(u, v) .
$$

We denote by $\langle\zeta, v\rangle, \zeta \in \overline{\mathcal{H}}, v \in \Omega$ the value of $\zeta$ at $v$. The $\mathbb{C}$-linear map $\phi_{E}: \Omega \rightarrow \overline{\mathcal{H}}$ such that $H(u, v)=\left\langle\phi_{E}(u), v\right\rangle$ induces a homomorphism, also denoted by $\phi_{E}$,

$$
\phi_{E}: \mathrm{J}(X) \rightarrow \widehat{\mathrm{J}(X)} \text {. }
$$

The matrix $-J=J^{-1}=\left(\begin{array}{cc}0 & -I \\ I & 0\end{array}\right)$ is the rational representation of $\phi_{E}$ with respect to the homology basis $\lambda_{1}, \ldots, \lambda_{2 g}$ for $H_{1}(X, \mathbb{Z})$ above and the dual basis $\alpha_{1}, \ldots, \alpha_{2 g}$ for $H^{1}(X, \mathbb{Z})$, i.e., $\alpha_{j}\left(\lambda_{k}\right)=\delta_{j k}$. Also, we can transport $E$ to $\overline{\mathcal{H}}$ by defining

$$
E^{\prime}\left(\phi_{E}(u), \phi_{E}(v)\right)=E(u, v) \text {. }
$$

We will obtain a bound for $\sharp \mathcal{I}(X)$ by counting the possible numbers of pull backs of holomorphic differentials. Thus, we will mainly deal with the dual Jacobian varieties and the endomorphisms of them. By an underlying real structure for a $g$-dimensional complex torus $T=V / \Gamma$, we mean the real torus $\mathbb{R}^{2 g} / \mathbb{Z}^{2 g}$ together with a map $\mathbb{R}^{2 g} / \mathbb{Z}^{2 g} \rightarrow T$ induced by a linear map $\mathbb{R}^{2 g} \ni x \mapsto \Pi x \in \mathbb{C}^{g}$ where $\Pi$ is a period matrix. For Jacobian varieties, it is known that the real part $E(i u, v)$ of the harmitian $H(u, v)$ is symmetric positive. Here, we define an inner product.

Definition. On $\mathbb{R}^{2 g}$, which is over the underlying real structure for $\widehat{\mathrm{J}(X)}$, we define an inner product by

$$
(x, y)_{X}=E^{\prime}(i \widehat{\Pi} x, \widehat{\Pi} y)
$$


where $\widehat{\Pi}$ is a period matrix for $\widehat{\mathrm{J}(X)}$. We define a norm $\|\cdot\|$ by

$$
\|x\|=\sqrt{(x, x)_{X}} .
$$

For an arbitrary $\mathfrak{g} \in \operatorname{End}(\mathrm{J}(X))$, put $\mathfrak{g}^{\prime}=\phi_{E}{ }^{-1} \circ{ }^{t} \mathfrak{g} \circ \phi_{E}$ and ${ }^{t} \mathfrak{g}^{\prime}=\phi_{E} \circ \mathfrak{g} \circ \phi_{E}{ }^{-1}$. (We observe $\left({ }^{t} \mathfrak{g}\right)^{\prime}={ }^{t}\left(\mathfrak{g}^{\prime}\right)$ holds.) This map ${ }^{\prime}: \operatorname{End}(\mathrm{J}(X)) \rightarrow \operatorname{End}(\mathrm{J}(X))$ (or $\operatorname{End}(\widehat{\mathrm{J}(X)}) \rightarrow \operatorname{End}(\widehat{\mathrm{J}(X)}))$ is so-called the Rosati involution. For a nonconstant holomorphic map $f: X \rightarrow Y$, denote by $\mathfrak{f}$ the induced homomorphism between the Jacobians. Put $\mathfrak{f}^{\prime}=\phi_{E}{ }^{-1} \circ{ }^{t} \mathfrak{f} \circ \phi_{E^{\prime}}$, where $\phi_{E^{\prime}}: \mathrm{J}(Y) \rightarrow \widehat{\mathrm{J}(Y)}$ is defined similar to $\phi_{E}: \mathrm{J}(X) \rightarrow \widehat{\mathrm{J}(X)}$. If we denote by $J^{\prime}=\left(\begin{array}{cc}0 & I \\ -I & 0\end{array}\right)$ where each entry is $\gamma \times \gamma$, then $-J^{\prime}=J^{\prime-1}$ is the rational representation of $\phi_{E^{\prime}}: \mathrm{J}(Y) \rightarrow \widehat{\mathrm{J}(Y)}$. We call $\mathfrak{F}=\mathfrak{f}^{\prime} \circ \mathfrak{f}(\in \operatorname{End}(\mathrm{J}(X)))$ the endomorphism associated with $f$.

Lemma 1. For an arbitrary $\mathfrak{g} \in \operatorname{End}(J(X))$, denoting by ${ }^{t} G$ and ${ }^{t} G^{\prime}$ the rational representations of the duals ${ }^{t} \mathfrak{g}$ and ${ }^{t} \mathfrak{g}^{\prime}$, respectively, we have

$$
\left({ }^{t} G x,{ }^{t} G y\right)_{X}=\left({ }^{t} G^{\prime t} G x, y\right)_{X}=\left(x,{ }^{t} G^{\prime t} G y\right)_{X},
$$

for all $x, y \in \mathbb{R}^{2 g}$. In particular, when $\mathfrak{F}$ is the endomorphism associated with some holomorphic map $f: X \rightarrow Y$ of degree $d$, denoting by $\mathcal{F}$ the rational representation of $\mathfrak{F}$, we have

$$
\left({ }^{t} \mathcal{F}(x),{ }^{t} \mathcal{F}(y)\right)_{X}=d\left({ }^{t} \mathcal{F}(x), y\right)_{X} .
$$

Proof. The proof of (2) is standard (see e.g. $[\mathrm{L}, \mathrm{L}-\mathrm{B}, \overline{\mathrm{Mm}}]$ ). For the homomorphism $\mathfrak{f}: J(X) \rightarrow J(Y)$ induced by $f: X \rightarrow Y, \mathfrak{f} \circ \mathfrak{f}^{\prime}$ is just multiplying $d$ on $\mathrm{J}(Y)$ (cf. [Mr2], [T1]). Thus, we have ${ }^{t} \mathcal{F}^{\prime t} \mathcal{F}=d^{t} \mathcal{F}$. This implies (3).

Lemma 2. Let $f: X \rightarrow Y$ be a nonconstant holomorphic map of degree $d$, and let $\mathfrak{F}$ be the endomorphism associated with $f$. Then, denoting by $\mathcal{F}$ the rational representation of $\mathfrak{F}$, we have

$$
\left\|^{t} \mathcal{F}(x)\right\| \leq d\|x\|
$$

for an arbitrary vector $x \in \mathbb{R}^{2 g}$.

Proof. The equality (3) and the Schwarz inequality imply (4).

Also, we give a lemma concerning holomorphic differentials. This is the key to reduce the bound.

Lemma 3. Let $f_{1}: X \rightarrow Y_{1}$ be a holomorphic map of degree $d$, and let $\mathfrak{f}_{1}: J(X) \rightarrow$ $J\left(Y_{1}\right)$ be the homomorphism induced by $f_{1}$. Take an arbitrary $u \in{ }^{t} \mathfrak{f}_{1}\left(\widehat{J\left(Y_{1}\right)}\right)$. Then, the number of isomorphic classes of holomorphic maps $f_{i}: X \rightarrow Y_{i}$ of degree $d$ such that the dual map ${ }^{t} \mathfrak{f}_{i}: \widehat{J\left(Y_{i}\right)} \rightarrow \widehat{J(X)}$ of the induced homomorphism $\mathfrak{f}_{i}$ satisfies $u \in{ }^{t} \mathfrak{f}_{i}\left(\widehat{J\left(Y_{i}\right)}\right)$ is at most $\left(\begin{array}{c}2 g-2 \\ d\end{array}\right) \times(2 g-1)^{d}$.

Proof. The assumption means that there exist holomorphic differentials $\phi_{1}$ on $Y_{1}$ and $\phi_{i}$ on $Y_{i}$ such that their pull backs satisfy $f_{1 *} \phi_{1}=f_{i *} \phi_{i}$.

Then, for a zero $p_{01}$ of $\phi_{1}$, the number of possible $f_{1}^{-1}\left(p_{01}\right)$ (counting multiplicities) that can occur is at most $\left(\begin{array}{c}2 g-2 \\ d\end{array}\right)$. After determining $\phi=f_{1 *} \phi_{1}$ and $f_{1}^{-1}\left(p_{01}\right)$, we can show that there are at most $(2 g-1)^{d}$ possible isomorphic classes of holomorphic maps of degree $d$ as follows. 
Let $f_{i}: X \rightarrow Y_{i}$ be holomorphic maps $(i=1,2)$. Suppose that there are holomorphic differentials $\phi_{1}$ and $\phi_{2}$ on $Y_{1}$ and $Y_{2}$, respectively, with $f_{1 *} \phi_{1}=f_{2 *} \phi_{2}$, and there is a zero $p_{01}$ (resp. $p_{02}$ ) of $\phi_{1}$ (resp. $\phi_{2}$ ) satisfying $f_{1}^{-1}\left(p_{01}\right)=f_{2}^{-1}\left(p_{02}\right)$. We put $\phi=f_{1 *} \phi_{1}=f_{2 *} \phi_{2}$. Let $\tilde{p}_{0} \in f_{1}^{-1}\left(p_{01}\right)=f_{2}^{-1}\left(p_{02}\right)$. Take a sufficiently small neighbourhood $U_{\tilde{p}_{0}}$ (resp. $U_{p_{0 i}}$ ) of $\tilde{p}_{0}$ (resp. $p_{0 i}$ ) so that there is no zero of $\phi\left(\right.$ resp. $\left.\phi_{i}\right)$ on $U_{\tilde{p}_{0}}\left(\right.$ resp. $\left.U_{p_{0 i}}\right)$ except $\tilde{p}_{0}\left(\right.$ resp. $\left.p_{0 i}\right)$, and that $f_{i}\left(U_{\tilde{p}_{0}}\right) \subset U_{p_{0 i}}(i=$ $1,2)$. We may take a local coordinate $z\left(\right.$ resp. $\left.z_{i}\right)$ on $U_{\tilde{p}_{0}}\left(\right.$ resp. $\left.U_{p_{0 i}}\right)$ such that $z\left(\tilde{p}_{0}\right)=0\left(\right.$ resp. $\left.z_{i}\left(p_{0 i}\right)=0\right)$ and the differential is written as

$$
\phi=z^{m} d z \quad\left(\text { resp. } \phi_{i}=z_{i}^{n_{i}} d z_{i}\right) .
$$

Recalling that $f_{1}^{-1}\left(p_{01}\right)=f_{2}^{-1}\left(p_{02}\right)$, we see $n_{1}=n_{2}$ and we will denote it by $n$ for brevity. We take two real lines $\gamma_{i}:[0, a) \rightarrow U_{p_{0 i}}$ with $\gamma_{i}(t)=t \in \mathbb{R}$ in the local coordinates $z_{i}(i=1,2)$. For an arbitrary $\tilde{p} \in U_{\tilde{p}_{0}} \backslash\left\{\tilde{p}_{0}\right\}$,

$$
\int_{0}^{\tilde{p}} z^{m} d z=\int_{0}^{f_{1}(\tilde{p})} z_{1}^{n} d z_{1}=\int_{0}^{f_{2}(\tilde{p})} z_{2}^{n} d z_{2}
$$

hence the number of possible positions for the set of lifts of $\gamma_{1}$ (thus also those of $\left.\gamma_{2}\right)$ in $U_{\tilde{p}_{0}}$ is at most $m+1 \leq 2 g-1$. Accordingly, the total number of possible positions for the set of all the lifts of $\gamma_{1}$ is at most $(2 g-1)^{d}$. Let $\left\{\tilde{p}_{0 j}\right\}_{j=1}^{N}=$ $f_{1}^{-1}\left(p_{01}\right)\left(=f_{2}^{-1}\left(p_{02}\right)\right)$. Suppose that, for every $\tilde{p}_{0 j} \in f_{1}^{-1}\left(p_{01}\right), U_{\tilde{p}_{0 j}} \cap f_{1}^{-1}\left(\gamma_{1}\right)=$ $U_{\tilde{p}_{0 j}} \cap f_{2}^{-1}\left(\gamma_{2}\right)$, that is, the set of lifts of $\gamma_{1}$ coincide with that of $\gamma_{2}$. Then, it is easy to see that we can define a local conformal map $h: f_{1}\left(U_{\tilde{p}_{0 j}}\right) \rightarrow f_{2}\left(U_{\tilde{p}_{0 j}}\right)$ such that

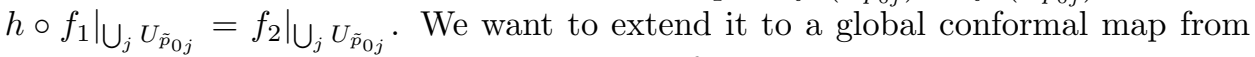
$Y_{1}$ to $Y_{2}$, and actually it is possible. Indeed, for an arbitrary point $p \in Y_{1}$, we will draw a curve $c$ from $p_{01}$ to $p$ avoiding branch points of $f_{1}$ other than possibly at $p_{01}$ and $p$. Let $\tilde{c}$ and $\tilde{c}^{\prime}$ be two lifts of $c$ by $f_{1}$. Then, we see that $f_{2}(\tilde{c})=f_{2}\left(\tilde{c}^{\prime}\right)$ since $h \circ f_{1}$ is well-defined near $\tilde{p}_{0 j}(j=1, \ldots, N)$. This implies that $h$ is well-defined on $Y_{1}$. It is easy to see that $h$ is invertible.

\section{A BOUND FOR $\mathcal{I}(X)$}

Now, we will recall the notion of successive minima which is a basic tool in the geometry of numbers (see e.g. [C]). In $n$-dimensional real vector space, let $\Lambda$ be a lattice, that is, the set of all points

$$
x=u_{1} a_{1}+\cdots+u_{n} a_{n} \in \Lambda
$$

with integers $u_{1}, \ldots, u_{n}$, and fixed linearly independent vectors $a_{1}, \ldots, a_{n}$. Let $F(x)$ be a distance function, namely, $F(x)$ is a non-negative and continuous function with $F(t x)=t F(x)(t \geq 0)$. The $k$-th successive minimum $\lambda_{k}$ of the distance function $F$ with respect to the lattice is the lower bound of the numbers $\lambda$ such that $\{\|x\|<\lambda\}$ contains $k$ linearly independent lattice points. In this paper, we take $\Lambda=\left\{x \in \mathbb{Z}^{2 g}\right\}$, and the distance function $F(x)$ is $\|x\|=\sqrt{(x, x)_{X}}$.

Notation. Let $a_{1}, \ldots, a_{2 g}$ be linearly independent points of the lattice such that $\left\|a_{k}\right\|=\lambda_{k}$ for $1 \leq k \leq 2 g$, where $\lambda_{k}$ is the $k$-th successive minimum.

We estimate the possible number of pull backs of an element in $H^{1}(X, \mathbb{Z})$, that is, the lattice of the dual Jacobian variety, to count the number of holomorphic maps from $X$. For this purpose, matrix representations are effective. In the following, 
rational representations of endomorphisms are considered to be matrix representations with respect to the homology basis such that the alternating form $E$ is expressed as $J=\left(\begin{array}{cc}0 & I \\ -I & 0\end{array}\right)$ with them.

Proposition. Let $f_{i}: X \rightarrow Y_{i}$ be nonconstant holomorphic maps, and $\mathcal{F}_{i}$ the rational representations of the endomorphisms associated with $f_{i}(i=1,2)$. Suppose that, for some $k<2 g$,

$$
\left\{\begin{array}{l}
{ }^{t} \mathcal{F}_{1} a_{1}=\cdots={ }^{t} \mathcal{F}_{1} a_{k-1}=0, \\
{ }^{t} \mathcal{F}_{2} a_{1}=\cdots={ }^{t} \mathcal{F}_{2} a_{k-1}=0,
\end{array}\right.
$$

and that there exists some integer $l>(2 g-2)^{2}$ such that ${ }^{t} \mathcal{F}_{1} a_{k} \equiv{ }^{t} \mathcal{F}_{2} a_{k}(\bmod . \quad l)$ holds. Then ${ }^{t} \mathcal{F}_{1} a_{k}={ }^{t} \mathcal{F}_{2} a_{k}$. If, in addition, $Y_{1}$ and $Y_{2}$ are of the same genus $\gamma$, then the assumption $l>(2 g-2)^{2}$ can be replaced by $l>(2 g-2)^{2} /(\gamma-1)^{2}$.

Remark. Here, $k$ may be equal to 1 . In this case, assumption (5) is not needed.

Proof. Let $D=\mathcal{F}_{1}-\mathcal{F}_{2}$. Then, $D$ is the rational representation of some endomorphism of $\mathrm{J}(X)$. It suffices to show that

$$
{ }^{t} D^{\prime t} D a_{k}=0 .
$$

Indeed, using formula (2) in Lemma 1, we have

$$
\left({ }^{t} D a_{k},{ }^{t} D a_{k}\right)_{X}=\left({ }^{t} D^{t} D a_{k}, a_{k}\right)_{X},
$$

and because of the property of inner products, (6) implies that ${ }^{t} D a_{k}=0$ which means ${ }^{t} \mathcal{F}_{1} a_{k}={ }^{t} \mathcal{F}_{2} a_{k}$.

To show (6), we estimate the norm of the vector ${ }^{t} D^{\prime t} D a_{k}$. First, we note that ${ }^{t} D^{\prime t} D x, a_{1}, \ldots, a_{k-1}$ are linearly independent for any vector $x \in \mathbb{R}^{2 g}$ if ${ }^{t} D^{\prime t} D x$ is not zero. Indeed, using (2) again, we see $\left({ }^{t} D^{\prime t} D x, a_{j}\right)_{X}=\left({ }^{t} D x,{ }^{t} D a_{j}\right)_{X}=0$ for $j=1, \ldots, k-1$ by the assumption. Thus, ${ }^{t} D^{\prime t} D x, a_{1}, \ldots, a_{k-1}$ are linearly independent. By the assumption ${ }^{t} D a_{k} \equiv 0(\bmod$. $l)$, the vector ${ }^{t} D^{\prime t} D a_{k}$ can be written in the form ${ }^{t} D^{\prime t} D a_{k}=l \times n$, where $n \in \mathbb{Z}^{2 g}$. Thus, if it is not 0 , then

$$
\left\|^{t} D^{\prime t} D a_{k}\right\| \geq l \lambda_{k},
$$

since the $n$ is a point of the lattice and not in the span of $\left\{a_{1}, \ldots, a_{k-1}\right\}$. Next, we will give an upper bound for $\left\|^{t} D^{\prime t} D a_{k}\right\|$. Set $\tilde{a}={ }^{t} D a_{k}$. By an easy calculation, we see that $\mathcal{F}_{i}^{\prime}=\mathcal{F}_{i}$ for $i=1,2$. Thus,

$$
\left\|{ }^{t} D^{\prime} \tilde{a}\right\| \leq\left\|{ }^{t} \mathcal{F}_{1} \tilde{a}\right\|+\left\|{ }^{t} \mathcal{F}_{2} \tilde{a}\right\| \leq d_{1}\|\tilde{a}\|+d_{2}\|\tilde{a}\|,
$$

where $d_{i}$ is the degree of $f_{i}(i=1,2)$. The first inequality is just the triangle inequality, and the second one is obtained by (4) in Lemma 2. Using Lemma 2 again, we have

$$
\|\tilde{a}\|=\left\|\left({ }^{t} \mathcal{F}_{1}-{ }^{t} \mathcal{F}_{2}\right) a_{k}\right\| \leq\left\|{ }^{t} \mathcal{F}_{1} a_{k}\right\|+\left\|{ }^{t} \mathcal{F}_{2} a_{k}\right\| \leq\left\|a_{k}\right\|\left(d_{1}+d_{2}\right) .
$$

Therefore, we have

$$
\left\|{ }^{t} D^{\prime t} D a_{k}\right\| \leq\left\|a_{k}\right\|\left(d_{1}+d_{2}\right)^{2}=\left(d_{1}+d_{2}\right)^{2} \lambda_{k} .
$$

By the Riemann-Hurwitz formula, $d_{i} \leq g-1$ and the right-hand side of (8) is $\leq 2^{2}(g-1)^{2} \lambda_{k}$. Using the inequality (7), we see that ${ }^{t} D^{\prime t} D a_{k}$ must be 0 since 
$l>2^{2}(g-1)^{2}$. If, in addition, $Y_{1}$ and $Y_{2}$ are of the same genus $\gamma$, then $d_{i} \leq$ $(g-1) /(\gamma-1)$ and the right-hand side of $(8)$ is $\leq 2^{2}(g-1)^{2} /(\gamma-1)^{2} \lambda_{k}$. Now, the proof is completed since (6) is shown.

We will say that $\mathcal{F}_{i} \in M(2 g, 2 g ; \mathbb{Z})$ is of the $k$-th type if ${ }^{t} \mathcal{F}_{1} a_{1}=\cdots={ }^{t} \mathcal{F}_{1} a_{k-1}=$ 0 and ${ }^{t} \mathcal{F} a_{k} \neq 0$.

Proof of the Theorem. To make the calculation easier, first, we fix the genus $\gamma$ of target surfaces and the degree $d$ of maps. Then we note that the number of possible types for associated endomorphisms $\mathcal{F}_{i}$ is at most $2 g-2 \gamma+1$ since the rank of each $\mathcal{F}_{i}$ is $2 \gamma$. For a fixed endomorphism $\mathcal{F}_{i}$ of $k$-th type, the number of possible $k$-th rows is at most $\left\{(2 g-2)^{2} /(\gamma-1)^{2}+1\right\}^{2 g}$ by the Proposition. If $\mathcal{F}_{1}$ and $\mathcal{F}_{2}$ have the same $k$-th row, then ${ }^{t} \mathcal{F}_{1}$ and ${ }^{t} \mathcal{F}_{2}$ have the same $k$-th column. Put $\mathfrak{f}_{i}: \mathrm{J}(X) \rightarrow \mathrm{J}\left(Y_{i}\right)$ the homomorphism induced by $f_{i}$ and $F_{i}$ the rational representation of $\mathfrak{f}_{i}(i=1,2)$. We can write ${ }^{t} \mathcal{F}_{i}={ }^{t} F_{i} J^{\prime-1} F_{i} J$ for $i=1,2$. Let $e_{k}$ be a vector in $\mathbb{R}^{2 g}$ whose $k$-th entry is 1 and others are zero. Then, we see ${ }^{t} F_{1} J^{\prime-1} F_{1} J e_{k}={ }^{t} F_{2} J^{\prime-1} F_{2} J e_{k}$ and this means $x_{i}:=J^{\prime-1} F_{i} J e_{k} \in H^{1}\left(Y_{i}, \mathbb{Z}\right)(i=1,2)$ satisfy ${ }^{t} F_{1} x_{1}={ }^{t} F_{2} x_{2}$. This implies that ${ }^{t} \mathfrak{f}_{1}\left(\widehat{\Pi}_{1} x_{1}\right)={ }^{t} \mathfrak{f}_{2}\left(\widehat{\Pi}_{2} x_{2}\right)$, where $\widehat{\Pi}_{1}$ (resp. $\left.\widehat{\Pi}_{2}\right)$ is the period matrix for $\widehat{\mathrm{J}\left(Y_{1}\right)}$ (resp. $\left.\widehat{\mathrm{J}\left(Y_{2}\right)}\right)$. Applying Lemma 3 , we see the number of all isomorphic classes of nonconstant holomorphic maps into compact Riemann surfaces of genus $\gamma(>1)$ smaller than $g$ satisfies,

$$
\begin{aligned}
& \sharp \mathcal{I}_{\gamma}(X)<\sum_{d>1}(2 g-2 \gamma+1) \times\left\{\left(\frac{2 g-2}{\gamma-1}\right)^{2}+1\right\}^{2 g} \times\left(\begin{array}{c}
2 g-2 \\
d
\end{array}\right) \times(2 g-1)^{d} \\
< & \left\{\left(\frac{2 g-2}{\gamma-1}\right)^{2}+1\right\}^{2 g} \times 2^{2 g-2} \times(2 g-1)^{\frac{g-1}{\gamma-1}} \times(2 g-2 \gamma+1)(g-\gamma) /(\gamma-1) .
\end{aligned}
$$

Now, we obtain (1):

$$
\sharp \mathcal{I}(X)=\sum_{g>\gamma>1} \sharp \mathcal{I}_{\gamma}(X)<(2 g)^{4 g} \times 2^{2 g-3} \times(2 g-1)^{g-1}(2 g-3)(g-2)(g-1) .
$$

\section{REFERENCES}

[A-P] Alzati, A., Pirola, G.P., Some remarks on the de Franchis theorem, Ann. Univ. Ferrara Sez. VII (N.S.) 36 (1990), 45-52. MR.1151481 (93b:14041)

[C] Cassels, J.W.S., An Introduction to the geometry of numbers, 2nd pr., Springer-Verlag, New York, Heidelberg, and Berlin, 1971. MR0306130 (46:5257)

[F] de Franchis, M., Un teorema sulle involuzioni irrazionali, Rend. Circ. Mat. Palermo 36 (1913), 368.

[H-S] Howard, A., Sommese, A. J., On the theorem of de Franchis, Ann. Scoula. Norm. Sup. Pisa Cl. Sci. (4) 10 (1983), 429-436. MR0739918 (85k:32048)

[K] Kani, E., Bounds on the number of non-rational subfields of a function field, Invent. Math. 85 (1986), 185-198. MF 0842053 (87i:14021)

[L] Lang, S., Abelian varieties, Springer-Verlag, New York, Heidelberg, and Berlin, 1983. MR0713430 (84g:14041)

[L-B] Lange, H., Birkenhake, Ch., Complex abelian varieties, Springer-Verlag, New York, Heidelberg, and Berlin, 1992. MF,1217487 (94j:14001)

[Mr1] Martens, H., Observations on morphisms of closed Riemann surfaces, Bull. London Math. Soc. 10 (1978), 209-212. MR0480985 (58:1132)

[Mr2] — Mappings of closed Riemann surfaces, Proc. Sympos. Pure Math. 49, Part 1 (1989), 531-539. MR.1013150(90i:14030)

[Mm] Mumford, D., Abelian varieties, Oxford University Press, London, 1970. MR0282985 $(44: 219)$ 
[T1] Tanabe, M., On rigidity of holomorphic maps of Riemann surfaces, Osaka J. Math. 33 (1996), 485-496. MR.1416060 (97i:32024)

[T2] $\longrightarrow$ A bound for the theorem of de Franchis, Proc. Amer. Math. Soc. 127 (1999), 2289-2295. MR1600153 (99j:30046)

Department of Mathematics, Tokyo Institute of Technology, Ohokayama, Meguro, TOKYO, 152-8551, JAPAN

E-mail address: tanabe@math.titech.ac.jp 